\title{
QCD at Finite Isospin Density
}

\author{
D.T. Son ${ }^{1,3}$ and M.A. Stephanov ${ }^{2,3}$ \\ 1 Physics Department, Columbia University, New York, New York 10027 \\ 2 Department of Physics, University of Illinois, Chicago, Illinois 6060\%-7059 \\ ${ }^{3}$ RIKEN-BNL Research Center, Brookhaven National Laboratory, Upton, New York 11973
}

(May 2000)

\begin{abstract}
QCD at finite isospin chemical potential $\mu_{I}$ has no fermion sign problem and can be studied on the lattice. We solve this theory analytically in two limits: at low $\mu_{I}$, where chiral perturbation theory is applicable, and at asymptotically high $\mu_{I}$, where perturbative QCD works. At low isospin density the ground state is a pion condensate, whereas at high density it is a Fermi liquid with Cooper pairing. The pairs carry the same quantum numbers as the pion. This leads us to conjecture that the transition from hadron to quark matter is smooth, which passes several tests. Our results imply a nontrivial phase diagram in the space of temperature and chemical potentials of isospin and baryon number.
\end{abstract}

Introduction.-Ample knowledge of $\mathrm{QCD}$ in the regime of finite temperature and baryon density is crucial for understanding a wide range of phenomena from heavy ion collisions to neutron stars and cosmology. Firstprinciples lattice numerical Monte Carlo calculations provide a solid basis for our knowledge of the finitetemperature regime. However, the regime of finite baryon chemical potential $\mu_{B}$ is still inaccessible by Monte Carlo because present methods of evaluating the QCD partition function require taking a path integral with a measure which includes a complex fermion determinant. Ignoring the determinant (as in the popular quenched approximation) leads to qualitatively wrong answers for finite $\mu_{B}$ [1]. Such a contrast to the case of $\mu_{B}=0$, where the quenched approximation proved useful, comes from the fact that the latter corresponds to an unphysical theory with pairs of quarks of opposite baryon charges (conjugate quarks) [2]. This is one of the main reasons why our understanding of QCD at finite baryon density is still rudimentary. Many interesting phenomena, such as color superconductivity and color-flavor locking [3], occur at finite baryon density, beyond the reach of current lattice techniques.

To understand the regime of finite baryon density one would need to follow the transition from hadronic to quark degrees of freedom by increasing the density of a conserved charge (such as baryon number), i.e., without invoking the temperature. This is the motivation for us to turn to QCD at finite chemical potential $\mu_{I}$ of isospin (more precisely, of the third component, $I_{3}$ ). Nature provides us with nonzero $\mu_{I}$ systems in the form of isospin-asymmetric matter. These always contain both isospin density and baryon density. In any realistic setting $\mu_{I} \ll \mu_{B}$. In this paper, however, we shall consider an idealization in which $\mu_{I}$ is nonzero while $\mu_{B}=0$. Such a system is unstable with respect to weak decays which do not conserve isospin. However, since we are interested in the dynamics of strong interaction alone, one can imagine that all relatively slow electroweak effects are turned off. Once this is done, we have a nontrivial regime which, as has been emphasized recently in [4], is accessible by present lattice Monte Carlo methods, while being, as we shall see, analytically tractable in various interesting limits. As a result, the system we consider has a potential to improve substantially our understanding of cold dense QCD. This regime carries many attractive traits of two-color QCD [5,6], but is realized in a physically relevant theory - QCD with three colors.

Positivity and QCD inequalities.-Since the Euclidean version of our theory has a real and positive fermion determinant, some rigorous results on the low-energy behavior can be obtained from QCD inequalities [7]6]. In vacuum $\mathrm{QCD}$, the latter rely on the following property of the Euclidean Dirac operator $\mathcal{D}=\gamma \cdot(\partial+i A)+m$ :

$$
\gamma_{5} \mathcal{D} \gamma_{5}=\mathcal{D}^{\dagger}
$$

which, in particular, implies positivity $\operatorname{det} \mathcal{D} \geq 0$. For the correlator of a generic meson $M=\bar{\psi} \Gamma \psi$, we can write, using (11) and the Schwartz inequality:

$$
\begin{aligned}
& \left\langle M(x) M^{\dagger}(0)\right\rangle_{\psi, A}=-\langle\operatorname{Tr} \mathcal{S}(x, 0) \Gamma \mathcal{S}(0, x) \bar{\Gamma}\rangle_{A}= \\
& \left\langle\operatorname{Tr} \mathcal{S}(x, 0) \Gamma i \gamma_{5} \mathcal{S}^{\dagger}(x, 0) i \gamma_{5} \bar{\Gamma}\right\rangle_{A} \leq\left\langle\operatorname{Tr} \mathcal{S}(x, 0) \mathcal{S}^{\dagger}(x, 0)\right\rangle_{A},
\end{aligned}
$$

where $\mathcal{S} \equiv \mathcal{D}^{-1}$ and $\bar{\Gamma} \equiv \gamma_{0} \Gamma^{\dagger} \gamma_{0}$. The inequality is saturated for mesons with $\Gamma=i \gamma_{5} \tau_{i}$, since $\mathcal{D}$ commutes with isospin $\tau_{i}$, which means that the pseudoscalar correlators are larger, point-by-point, than all other $I=1$ meson correlators [8]. As a consequence, one obtains an important restriction on the pattern of the symmetry breaking: for example, it cannot be driven by a condensate of $\left\langle\bar{\psi} \gamma_{5} \psi\right\rangle$, which would give $0^{+}$Goldstones.

At finite isospin density, $\mu_{I} \neq 0$, positivity still holds [14 and certain inequalities can be derived (in contrast with the case of $\mu_{B} \neq 0$ when there is no positivity). Now $\mathcal{D}=\gamma \cdot(\partial+i A)+\frac{1}{2} \mu_{I} \gamma_{0} \tau_{3}+m$, and Eq. (11) is not true anymore, since the operation on the right-hand side of (1) changes the relative sign of $\mu_{I}$. However, provided $m_{u}=$ $m_{d}$, interchanging up and down quarks compensates for 
this sign change (the $u$ and $d$ quarks play the role of mutually conjugate quarks (2]), i.e,

$$
\tau_{1} \gamma_{5} \mathcal{D} \gamma_{5} \tau_{1}=\mathcal{D}^{\dagger}
$$

Instead of isospin $\tau_{1}$ in (3) one can also use $\tau_{2}$ (but not $\tau_{3}$ ). Equation (3), in place of the now invalid Eq. (1), ensures that $\operatorname{det} \mathcal{D} \geq 0$. Repeating the derivation of the QCD inequalities, by using (3) we find that the lightest meson, or the condensate, must be in channels $\bar{\psi} i \gamma_{5} \tau_{1,2} \psi$, i.e., a linear combination of $\pi^{-} \sim \bar{u} \gamma_{5} d$ and $\pi^{+} \sim \bar{d} \gamma_{5} u$ states. Indeed, as shown below, in the two analytically tractable regimes of small and large $\mu_{I}$ the lightest mode is a massless Goldstone which is a linear combination of $\bar{u} \gamma_{5} d$ and $\bar{d} \gamma_{5} u$.

Small isospin densities.-When $\mu_{I}$ is small compared to the chiral scale (taken here to be $m_{\rho}$ ), we can use chiral perturbation theory. For zero quark mass and zero $\mu_{I}$ the pions are massless Goldstones of the spontaneously broken $\mathrm{SU}(2)_{L} \times \mathrm{SU}(2)_{R}$ chiral symmetry. If the quarks have small equal masses, the symmetry is only $\mathrm{SU}(2)_{L+R}$. The low-energy dynamics is governed by the familiar chiral Lagrangian for the pion field $\Sigma \in$ $\mathrm{SU}(2): \mathcal{L}=\frac{1}{4} f_{\pi}^{2} \operatorname{Tr}\left[\partial_{\mu} \Sigma \partial_{\mu} \Sigma^{\dagger}-2 m_{\pi}^{2} \operatorname{Re} \Sigma\right]$, which contains the pion decay constant $f_{\pi}$ and vacuum pion mass $m_{\pi}$ as phenomenological parameters. The isospin chemical potential further breaks $\mathrm{SU}(2)_{L+R}$ down to $\mathrm{U}(1)_{L+R}$. Its effect can be included to leading order in $\mu_{I}$ without additional phenomenological parameters by promoting $\mathrm{SU}(2)_{L} \times \mathrm{SU}(2)_{R}$ to a local gauge symmetry and viewing $\mu_{I}$ as the zeroth component of a gauge potential [6]. Gauge invariance thus fixes the way $\mu_{I}$ enters the chiral Lagrangian:

$$
\mathcal{L}_{\text {eff }}=\frac{f_{\pi}^{2}}{4} \operatorname{Tr} \nabla_{\nu} \Sigma \nabla_{\nu} \Sigma^{\dagger}-\frac{m_{\pi}^{2} f_{\pi}^{2}}{2} \operatorname{Re} \operatorname{Tr} \Sigma,
$$

where the covariant derivative is defined as

$$
\nabla_{0} \Sigma=\partial_{0} \Sigma-\frac{\mu_{I}}{2}\left(\tau_{3} \Sigma-\Sigma \tau_{3}\right)
$$

By using (何), it is straightforward to determine vacuum alignment of $\Sigma$ as a function of $\mu_{I}$ and the spectrum of excitations around the vacuum. We are interested in negative $\mu_{I}$, which favors neutrons over protons, as in neutron stars. The results are very similar to two-color QCD at finite baryon density [6]:

(i) For $\left|\mu_{I}\right|<m_{\pi}$, the system is in the same ground state as at $\mu_{I}=0: \bar{\Sigma}=1$. This is because the lowest lying pion state costs a positive energy $m_{\pi}-\left|\mu_{I}\right|$ to excite, which is impossible at zero temperature.

(ii) When $\left|\mu_{I}\right|$ exceeds $m_{\pi}$ it is favorable to excite $\pi^{-}$ quanta, which form a Bose condensate. In the language of the effective theory, such a pion condensate is described by a tilt of the chiral condensate $\bar{\Sigma}$,

$$
\begin{aligned}
\bar{\Sigma}= & \cos \alpha+i\left(\tau_{1} \cos \phi+\tau_{2} \sin \phi\right) \sin \alpha, \\
& \cos \alpha=m_{\pi}^{2} / \mu_{I}^{2} .
\end{aligned}
$$

The tilt angle $\alpha$ is determined by minimizing the vacuum energy. The energy is degenerate with respect to the angle $\phi$, corresponding to the spontaneous breaking of the $\mathrm{U}(1)_{L+R}$ symmetry generated by $I_{3}$ in the Lagrangian (4). The ground state is a pion superfluid, with one massless Goldstone mode. Since we start from a theory with three pions, there are two massive modes which can be identified with $\pi_{0}$ and a linear combination of $\pi^{+}$ and $\pi^{-}$. At the condensation threshold, $m_{\pi_{0}}=m_{\pi}$ and the mass of the other mode is $2 m_{\pi}$, while for $\left|\mu_{I}\right| \gg m_{\pi}$ both masses approach $\left|\mu_{I}\right|$.

The isospin density is found by differentiating the ground state energy with respect to $\mu_{I}$ and is equal to:

$$
n_{I}=f_{\pi}^{2} \mu_{I} \sin ^{2} \alpha=f_{\pi}^{2} \mu_{I}\left(1-\frac{m_{\pi}^{4}}{\mu_{I}^{4}}\right), \quad\left|\mu_{I}\right|>m_{\pi} .
$$

For $\left|\mu_{I}\right|$ just above the condensation threshold, $\left|\mu_{I}\right|-$ $m_{\pi} \ll m_{\pi}$, Eq. (7) reproduces the equation of state of the dilute nonrelativistic pion gas [6].

It is also possible to find baryon masses, i.e., the energy cost of introducing a single baryon into the system. The most interesting baryons are those with lowest energy and highest isospin, i.e. neutron $n$ and $\Delta^{-}$isobar. There are two effects of $\mu_{I}$ on the baryon masses. The first comes from the isospin of the baryons, which effectively reduces the neutron mass by $\frac{1}{2}\left|\mu_{I}\right|$ and the $\Delta^{-}$ mass by $\frac{3}{2}\left|\mu_{I}\right|$. Alone, this effect would lead to the formation of baryon/antibaryon Fermi surfaces, manifested in nonvanishing zero-temperature baryon susceptibility $\chi_{B} \equiv \partial n_{B} / \partial \mu_{B}$ when $\mu_{I}>\frac{2}{3} m_{\Delta}$. However, long before that, another effect turns on: the $\pi^{-}$'s in the condensate tend to repel the baryons, lifting up their masses. These effects can be treated in the framework of the baryon chiral perturbation theory [9], giving

$$
m_{n}=m_{N}-\frac{\left|\mu_{I}\right|}{2} \cos \alpha, \quad m_{\Delta^{-}}=m_{\Delta}-\frac{3\left|\mu_{I}\right|}{2} \cos \alpha
$$

in the approximation of nonrelativistic baryons. Equation (8) can be interpreted as follows: as a result of the rotation (6) of the chiral condensate, the nucleon mass eigenstate becomes a superposition of vacuum $n$ and $p$ states. The expectation value of the isospin in this state is proportional to $\cos \alpha$ appearing in (8). With $\cos \alpha$ given in Eq.(6), we see that the two mentioned effects cancel each other when $m_{\pi} \ll\left|\mu_{I}\right| \ll m_{\rho}$. Thus the baryon mass never drops to zero, and $\chi_{B}=0$ at zero temperature in the region of applicability of the chiral Lagrangian.

As one forces more pions into the condensate, the pions are packed closer and their interaction becomes stronger. When $\mu_{I} \sim m_{\rho}$, the chiral perturbation theory breaks down. To find the equation of state in this regime, full QCD has to be employed. As we have seen, this can be done using present lattice techniques since the fermion sign problem is not present at finite $\mu_{I}$, similar to the two-color QCD [5]. 
Asymptotically high isospin densities.-In the opposite limit of very large isospin densities, or $\left|\mu_{I}\right| \gg m_{\rho}$, the description in terms of quark degrees of freedom applies since the latter are weakly interacting due to asymptotic freedom. In our case of large negative $\mu_{I}$, or $n_{I}$, the ground state consists of $d$ quarks and $\bar{u}$ antiquarks which, neglecting the interaction, fill two Fermi spheres with equal radii $\left|\mu_{I}\right| / 2$. Turning on the interaction between the fermions leads to the instability with respect to the formation and condensation of Cooper pairs, similar, to some extent, to the diquark pairing at high baryon density [3]. In our case, $\mu_{I}<0$, the Cooper pair consists of a $\bar{u}$ and a $d$ in the color singlet channel. The order parameter has the same quantum numbers as the pion condensate at lower densities,

$$
\left\langle\bar{u} \gamma^{5} d\right\rangle \neq 0
$$

Because of Cooper pairing, the fermion spectrum acquires a gap $\Delta$ at the Fermi surface, where

$$
\Delta=b\left|\mu_{I}\right| g^{-5} e^{-c / g}, \quad c=3 \pi^{2} / 2
$$

where $g$ should be evaluated at the scale $\left|\mu_{I}\right|$. This behavior comes from the long-range magnetic interaction, as in the superconducting gap at large $\mu_{B}$ [10. The constant $c$ is smaller by a factor of $\sqrt{2}$ compared to the latter case due to the stronger one-gluon attraction in the singlet $q \bar{q}$ channel compared to the $\overline{\mathbf{3}}$ diquark channel. Consequently, the gap (10) is exponentially larger than the diquark gap at comparable baryon chemical potentials. By using the methods of [11], one can estimate $b \approx 10^{4}$.

The perturbative one-gluon exchange responsible for pairing at large $\mu_{I}$ does not distinguish $\bar{u} d$ and $\bar{u} \gamma_{5} d$ channels: the attraction is the same in both. The $\bar{u} \gamma_{5} d$ channel is favored by the instanton-induced interactions, which explains the fact that the condensate is a pseudoscalar and breaks parity. This is consistent with our observation that QCD inequalities also constrain the $I=1$ condensate to be a pseudoscalar at any $\mu_{I}$.

Quark-hadron continuity.-Since the order parameter (9) has the same quantum numbers and breaks the same symmetry as the pion condensate in the low-density regime, it is plausible that there is no phase transition along the $\mu_{I}$ axis. In this case the Bose condensate of weakly interacting pions smoothly transforms into the superfluid state of $\bar{u} d$ Cooper pairs. The situation is very similar to that of strongly coupled superconductors with a "pseudogap" [12, and possibly of high-temperature superconductors 13. This also parallels the continuity between nuclear and quark matter in three-flavor QCD as conjectured by Schäfer and Wilczek 14. We hence conjecture that, in two-flavor QCD, one can move continuously from the hadron phase to the quark phase without encountering a phase transition. Since a first order deconfinement phase transition at intermediate isospin chemical potential cannot be rigorously ruled out (though it is unlikely, see below), this conjecture needs to be verified by lattice calculations.

A number of nontrivial arguments support the continuity hypothesis. One notices that all fermions have a gap at large $\left|\mu_{I}\right|$, which implies that $\chi_{B}=0$ at $T=0$. This is also true at small $\mu_{I}$. It is thus natural to expect that $\chi_{B}$ remains zero at $T=0$ for all $\mu_{I}$, which also suggests one way to check the continuity on the lattice.

Another argument comes from considering the limit of a large number of colors $N_{c}$. In finite-temperature QCD, the fact that the number of gluon degrees of freedom is $\mathcal{O}\left(N_{c}^{2}\right)$ while that of hadrons is $\mathcal{O}\left(N_{c}^{0}\right)$ hints at a first order confinement-deconfinement phase transition. At very large $\mu_{I}$ thermodynamic quantities such as the density of isospin $n_{I}$ are proportional to $N_{c}$. On the other hand, in the large $N_{c}$ limit the pion decay constant scales as $f_{\pi}^{2}=\mathcal{O}\left(N_{c}\right)$, and according to Eq. (7) the isospin density in the pion gas is also proportional to $N_{c}$. Physically, the repulsion between pions becomes weaker as one goes to large $N_{c}$, thus more pions are stacked at a given chemical potential. As a result, the $N_{c}$ dependence of thermodynamic quantities is the same in the quark and the hadronic regimes.

Confinement. - At large $\mu_{I}$, gluons softer than $\Delta$ are not screened by the Meissner or by the Debye effect [15]: the condensate does not break gauge symmetry (in contrast to the color superconducting condensate [3]) and there are no low-lying color excitations to screen the electric field. Thus, the gluon sector below the $\Delta$ scale is described by pure gluodynamics, which is confining. This means there are no quark excitations above the ground state: all particles and holes must be confined in colorless objects, mesons and baryons, just like in vacuum QCD. If there is no transition along the $\mu_{I}$ axis, we expect confinement at all values of $\mu_{I}$. Since the running strong coupling $\alpha_{s}$ at the scale of $\Delta$ is small, the confinement scale $\Lambda_{\mathrm{QCD}}^{\prime}$ (which is, in general, different from $\Lambda_{\mathrm{QCD}}$ ) is much less than $\Delta$. At large $\mu_{I}$, we thus predict a temperature driven deconfinement phase transition at a temperature $T_{c}^{\prime}$ of order $\Lambda_{\mathrm{QCD}}^{\prime}$, which is expected to be of first order as in pure gluodynamics. Since $\Lambda_{\mathrm{QCD}}^{\prime} \ll \Delta$ the hadronic spectrum is similar to that of a heavy quarkonium, with $\Delta$ playing the role of the heavy quark mass.

The $\left(T, \mu_{I}\right)$ phase diagram.-By considering nonzero $\mu_{I}$, we make the phase diagram of QCD three dimensional: $\left(T, \mu_{B}, \mu_{I}\right)$. Two planes in this three-dimensonal space are of a special interest: the $\mu_{B}=0\left(T, \mu_{I}\right)$ plane, which is completely accessible by present lattice techniques, and the $T=0\left(\mu_{I}, \mu_{B}\right)$ plane, where the neutron star matter belongs. Two phenomena determine the $\left(T, \mu_{I}\right)$ phase plane (Fig.11): pion condensation and confinement. 


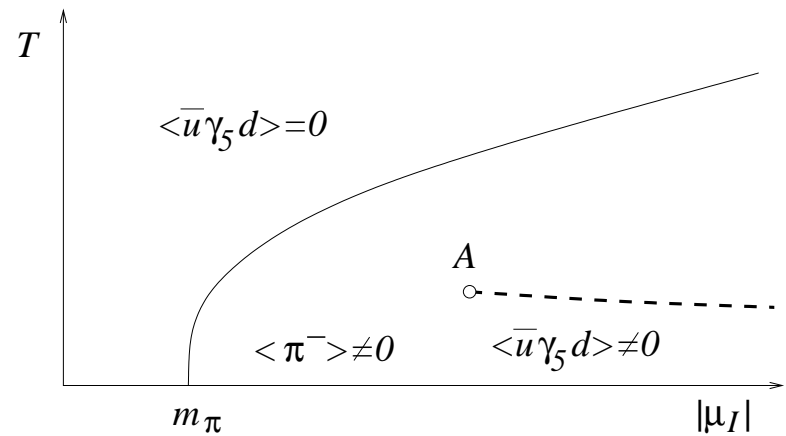

FIG. 1. Phase diagram of QCD at finite isospin density.

At sufficiently high temperature the condensate (9) melts (solid line in Fig. 1). For large $\mu_{I}$, this critical temperature is proportional to the BCS gap (10). There are two phases which differ by symmetry: the high temperature phase, where the explicit flavor $U(1)_{L+R}$ symmetry is restored, and the low-temperature phase, where this symmetry is spontaneously broken. The phase transition is in the $\mathrm{O}(2)$ universality class [16]. The critical temperature $T_{c}$ vanishes at $\mu_{I}=m_{\pi}$ and is an increasing function of $\mu_{I}$ in both regimes we studied: $\left|\mu_{I}\right| \ll m_{\rho}$ and $\left|\mu_{I}\right| \gg \Lambda_{\mathrm{QCD}}$. Thus, it is likely that $T_{c}\left(\mu_{I}\right)$ is a monotonous function of $\mu_{I}$. In addition, at large $\mu_{I}$, there is a first order deconfinement phase transition at $T_{c}^{\prime}$ much lower than $T_{c}\left(\mu_{I}\right)$. Since there is no phase transition at $\mu_{I}=0$ (for small $m_{u, d}$ ) or at $T=0$ (assuming quark-hadron continuity), this first-order line must end at some point $A$ on the $\left(T, \mu_{I}\right)$ plane (Fig. 1). The exact location of $A$ should be determined by lattice calculations; one of the possibilities is drawn in Fig. 1.

The $\left(\mu_{I}, \mu_{B}\right)$ phase diagram.-This phase diagram deserves a separate study. Here we shall only consider the regime $\left|\mu_{I}\right| \gg \mu_{B}$ (the opposite limit $\mu_{B} \gg\left|\mu_{I}\right|$ was considered in Ref. [17). Finite $\mu_{B}$ provides a mismatch between $\bar{u}$ and $d$ Fermi spheres, which makes the superconducting state unfavorable at some value of $\mu_{B}$ of order $\Delta$. It is known 18 that the destruction of this state occurs through two phase transitions: one at $\mu_{B}$ slightly below $\Delta / \sqrt{2}$ and another at $\mu_{B}=0.754 \Delta$. The ground state between the two phase transitions is the Fulde-FerrellLarkin-Ovchinnikov (FFLO) state [18], characterized by a spatially modulated superfluid order parameter $\left\langle\bar{u} \gamma_{5} d\right\rangle$ with a wavenumber of order $2 \mu_{B}$. The FFLO state has the same symmetries as the inhomogeneous pion condensation state which might form in electrically neutral nuclear matter at high densities 19]. It is conceivable that the two phases are actually one, i.e., continuously connected on the $\left(\mu_{I}, \mu_{B}\right)$ phase diagram.

The authors thank L. McLerran, J. Kogut, R. Pisarski, and E. Shuryak for discussions, the DOE Institute for Nuclear Theory at the University of Washington for its hospitality, and K. Rajagopal for drawing their attention to Ref. [18].
[1] I. Barbour et al, Nucl. Phys. B275 [FS17], 296 (1986); J.B. Kogut, M. P. Lombardo and D.K. Sinclair, Phys. Rev. D 51, 1282 (1995); Nucl. Phys. B, Proc. Suppl. 42, 514 (1995).

[2] M.A. Stephanov, Phys. Rev. Lett. 76, 4472 (1996).

[3] B.C. Barrois, PhD Thesis, California Institute of Technology, 1979; D. Bailin and A. Love, Phys. Rep. 107, 325 (1984); M. Alford, K. Rajagopal and F. Wilczek, Phys. Lett. B422, 247 (1998), Nucl. Phys. B537, 443 (1999); R. Rapp, T. Schäfer, E.V. Shuryak and M. Velkovsky, Phys. Rev. Lett. 81, 53 (1998); Ann. Phys. (N.Y.) 280, 35 (2000).

[4] M. Alford, A. Kapustin and F. Wilczek, Phys. Rev. D 59, 054502 (1999).

[5] E. Dagotto, F. Karsch, and A. Moreo, Phys. Lett. 169B, 421 (1986); E. Dagotto, A. Moreo, and U. Wolff, Phys. Rev. Lett. 57, 1292 (1986); Phys. Lett. B 186, 395 (1987); S. Hands, J.B. Kogut, M.-P. Lombardo, S.E. Morrison, Nucl. Phys. B558, 327 (1999); S. Hands and S.E. Morrison, hep-lat/9902012; hep-lat/9905021.

[6] J.B. Kogut, M.A. Stephanov, and D. Toublan, Phys. Lett. B 464, 183 (1999); J.B. Kogut, M.A. Stephanov, D. Toublan, J.J. Verbaarschot and A. Zhitnitsky, Nucl. Phys. B582, 477 (2000).

[7] D. Weingarten, Phys. Rev. Lett. 51, 1830 (1983); E. Witten, Phys. Rev. Lett. 51, 2351 (1983); S. Nussinov, Phys. Rev. Lett. 52, 966 (1984); D. Espriu, M. Gross and J.F. Wheater, Phys. Lett. 146B, 67 (1984).

[8] It is important, as is the case for $I=1$, that there is no disconnected piece after $\psi$ integration in (2). The proof does not apply, for example, to $\sigma$ or $\eta$ meson correlators, $\Gamma=1, \gamma_{5}$.

[9] See, e.g., H. Georgi, Weak Interaction and Modern Particle Theory (Benjamin-Cummings, Menlo Park, 1984).

[10] D.T. Son, Phys. Rev. D 59, 094019 (1999).

[11] W.E. Brown, J.T. Liu, and H.-C. Ren, Phys. Rev. D 61, 114012 (2000); 62, 054016 (2000); 62, 054013 (2000).

[12] A.J. Leggett, J. de Phys. 41, C7-19 (1980); P. Nozières and S. Schmitt-Rink, J. Low Temp. Phys. 59, 195 (1985).

[13] See, e.g., M. Randeria, cond-mat/9710223 and references therein.

[14] T. Schäfer and F. Wilczek, Phys. Rev. Lett. 82, 3956 (1999).

[15] This is similar to the behavior of the unbroken $\mathrm{SU}(2)_{c}$ sector of two-flavor color superconductors (see D.H. Rischke, Phys. Rev. D 62, 034007 (2000)).

[16] The width of the Ginzburg region is suppressed by $\left(\Delta / \mu_{I}\right)^{4}$ at large $\mu_{I}$ and also by $1 / N_{c}^{2}$ at large $N_{c}$ : J.B. Kogut, M.A. Stephanov and C.G. Strouthos, Phys. Rev. D 58, 096001 (1998).

[17] M. Alford, J. Bowers and K. Rajagopal, hep-ph/0008208.

[18] P. Fulde and A. Ferrell, Phys. Rev. 135, A550 (1964); A.I. Larkin and Yu.N. Ovchinnikov, Sov. Phys. JETP 20, 762 (1965); see also Ref. 17].

[19] A.B. Migdal, Sov. Phys. JETP 36, 1052 (1973); R.F. Sawyer, Phys. Rev. Lett. 29, 382 (1972); D.J. Scalapino, Phys. Rev. Lett. 29, 386 (1972). 